\title{
Korean Translation of the GRADE Series Published in the BMJ, 'GRADE: Going from Evidence to Recommendations' (A Secondary Publication)
}

\author{
Translated by: Eu Chang Hwang, Do Kyung Kim ${ }^{1}$, Ho Won Kang ${ }^{2}$, Ja Yoon Ku ${ }^{3}$, Hyun Jin Jung ${ }^{4}$, Hong Wook Kim ${ }^{5}$, \\ Jae Hung Jung ${ }^{6,7}$; Guideline Development Committee in the Korean Association of Urogenital Tract Infection and Inflammation \\ Department of Urology, Chonnam National University Hwasun Hospital, Chonnam National University Medical School, Hwasun, ${ }^{1}$ Department \\ of Urology, Soonchunhyang University Seoul Hospital, Soonchunhyang University College of Medicine, Seoul, ${ }^{2}$ Department of Urology, \\ Chungbuk National University College of Medicine, Cheongju, ${ }^{3}$ Department of Urology, Pusan National University Hospital, Busan, ${ }^{4}$ Department \\ of Urology, Daegu Catholic University School of Medicine, Daegu, ${ }^{5}$ Department of Urology, College of Medicine, Konyang University, \\ Daejeon, ${ }^{6}$ Department of Urology, ${ }^{7}$ Institute of Evidence Based Medicine, Yonsei University Wonju College of Medicine, Wonju, Korea
}

This article is the third translation of a GRADE series published in the BMJ for developing and presenting recommendations for managing patients. The strength of a recommendation reflects the extent to which we can be confident that desirable effects of an intervention outweigh any undesirable effects. GRADE classifies the strength of recommendations as strong or weak. The strength of recommendation is determined by the balance between desirable and undesirable consequences of alternative management strategies, quality of the evidence, variability in values and preferences, and the appropriate usage of resources.

Copyright (c) 2019, Korean Association of Urogenital Tract Infection and Inflammation. All rights reserved. (1) (\$) This is an open access article distributed under the terms of the Creative Commons Attribution (c) (1) () NC Non-Commercial License (http://creativecommons.org/licenses/by-nc/4.0) which permits unrestricted non-commercial use, distribution, and reproduction in any medium, provided the original work is properly cited.
Received: 18 September, 2019

Revised: 18 September, 2019

Accepted: 18 September, 2019

\section{Correspondence to: Jae Hung Jung}

(iD) https://orcid.org/0000-0002-4990-7098

Department of Urology, Yonsei University Wonju

College of Medicine, 20 Ilsan-ro, Wonju 26426, Korea

Tel: +82-33-741-0652, Fax: +82-33-741-1930

E-mail: geneuro95@yonsei.ac.kr

This article is the secondary publication (complete translation in Korean) of the article originally published in the $B M J$ in English (GRADE: going from evidence to recommendations. 2008;336:1049-51). The Editor-in-Chief of Urogenital Tract Infection decided to publish this secondary publication for the reader's sake, and it was approved by $B M J$. BMJ Publishing Group take no responsibility for the accuracy of the translation from the original published English language and are not liable for any errors that may have occurred.

\section{GRADE (Grading of Recommendations, As- sessment, Development and Evaluation): 근거에서 권고로}

\section{GRADE는 진료지침에서 권고를 '강함' 또는 '약 함' 으로 분류합니다. 이 논문을 통하여 이러한 권 고가 환자, 임상의사 및 정책결정자에게 지니는 함의를 설명하고자 합니다.}

본 논문은 GRADE 방법론에 대한 세 번째 시리즈로 $\mathrm{GRADE}$ 을 이용한 환자 진료 권고안 개발과정을 설명하고 임상의사에게 권고의 강도를 어떻게 해석하여야 하는지에 대하여 알아보고자 합니다[1].

\section{권고의 강도(Strength of a Recommendation) 는 무엇을 의미하는가?}

권고의 강도는 중재(intervention)의 바람직한 효과(desirable effects)가 바람직하지 않은 효과(undesirable effects)보다 어느 정도 더 크다고 확신할 수 있는지를 반영합니 다. 중재의 바람직한 효과로는 이환율(morbidity) 및 사망률 의 감소, 삶의 질의 증가, 치료 부담(burden of treatment: 예를 들어 치료 약물 복용 및 혈액검사의 불편함)의 감소, 그리고 치료 비용의 감소 등이 있습니다. 바람직하지 않은 효과로는 이환율, 사망률에 영향을 미치는 부작용 혹은 삶의 질 악화 그리고 치료 비용 상승을 포함합니다.

권고 강도를 결정하는 이전의 방법론들은 최대 9개 범주의 권고 강도를 사용했습니다[2]. GRADE는 강한 권고(strong 
recommendation)와 약한 권고(weak recommendation) 두 가지 범주로만 권고 강도를 분류합니다. 진료지침 개발 패널은 두 범주의 강도를 특정 짓기 위해 다른 단어를 선택할 수 있습니다. GRADE를 사용할 때 패널은 치료의 바람직한 효과가 바람직하지 않은 효과보다 크다고 확신할 때 권고를 '강함'으로 제시합니다. 약한 권고는 치료의 바람직한 효과가 바람직하지 않은 효과보다 클 수 있지만 진료지침 개발 패널이 이 효과에 대해 덜 확신한다는 것을 나타냅니다.

\section{강한 권고, 약한 권고는 구체적인 지침을 제공합 니다.}

$\mathrm{GRADE}$ 의 권고 강도에 대한 이분법적인 분류는 환자, 임상 의사, 그리고 정책결정자에 명확한 방향을 제공합니다.

강한 권고의 의미:

- 환자 입장: 해당 상황에서 대부분의 환자는 권고되는 중재를 원할 것이고 일부에서 원하지 않을 수 있음. 중재 가 제공되지 않을 경우 이에 대한 충분한 논의가 필요함. - 임상의사 입장: 대부분의 환자는 권고되는 치료를 받아야 함.

- 정책결정자 입장: 대부분의 경우 권고는 정책에 수용될 수 있음.

약한 권고의 의미:

- 환자 입장: 해당 상황에서 상당수의 환자는 권고되는 중재를 원하나 다른 상당수는 권고되는 치료를 원하지 않을 수 있음.

- 임상의사 입장: 각각의 환자에게 각기 다른 중재가 적합할 수 있음을 반드시 숙지 해야 함. 치료를 결정할 때 환자가 자신의 가치(values)와 선호도(preference)에 일치하는 결정을 할 수 있도록 도와주어야 함.

- 정책결정자 입장: 정책결정 시 이해당사자들의 충분한 토의와 참여가 필요함.

임상의사는 중재에 대하여 환자의 가치와 선호도가 다양할 수 있음을 고려하여야 합니다[3]. 강한 권고는 필요한 치료에 대하여 거의 모든 환자가 같은 선택을 할 것이기 때문에 임상의
사의 의학적 도움이 불필요할 수 있습니다. 약한 권고는 의사결 정에 도움이 필요할 수 있습니다.

보건 의료 관리자들(managers of healthcare systems) 은 의료의 질(quality of care) 관리에 관심을 기울이고 있습 니다. 진료지침은 관리자들에게 양질의 근거에 기반한 임상진 료(practice)와 전문가 의견과 관련된 임상진료를 구별하는 데 도움을 줄 수 있습니다. GRADE는 이것과 관련하여 명확한 지침을 제공합니다. 강한 권고와 관련된 중재들은 양질의 의료 로 고려될 수 있으며 약한 권고 경우에는 환자와 보호자와 대체 치료(alternative management strategies)에 대한 상 의가 양질의 의료를 제공하기 위하여 필요할 수 있습니다.

\section{권고 강도 결정을 위한 네 가지 핵심 요소}

권고 강도를 결정하는 첫 번째 핵심 요소는 중재의 바람직한 효과와 바람직하지 않은 효과의 차이입니다(Table 1 and Appendix 1) [1]. 예를 들어 미숙아를 출산할 예정인 여성에 게 산전 스테로이드의 사용은 유아호흡곤란증후군의 위험을 줄일 수 있습니다. 약물의 투여에 따르는 부작용은 적고, 환자 불편감이 적으며, 비용이 적게 들어 약물 사용의 장점이 단점보 다 횔씬 크기 때문에 강한 권고가 적절할 것입니다. 장점과 단점의 차이가 적은 경우에는 약한 권고가 보다 바람직할 것입니다. 다른 예로 뇌졸중 저위험군으로 심방세동이 있는 환자에서 warfarin은 뇌졸중의 위험도를 더 낮출 것이지만 약물 투여의 불편함과 더불어 출혈의 위험도가 증가할 수 있습니다. 이 경우 warfarin 치료는 환자의 가치와 선호도에 따라 달라질 수 있습니다.

두 번째 핵심 요소는 근거수준(quality of evidence)입니 다. 만약 우리가 중재의 이점(benefit)과 위해(harm)의 크기 (magnitude)에 대해 확신할 수 없다면 강한 권고를 하기는 어려울 것입니다. 예를 들어 장거리 비행기 여행을 하는 사람들 에게 압박스타킹을 사용하는 것은 명백히 심부정맥혈전증을 줄이는 데 도움을 줄 수 있으나 이러한 효과를 보고한 무작위 임상시험은 심각한 연구 비뚤림(눈가림 실패, 부정확한 결과 측정 방법)을 수반하고 있습니다. 결국 명백한 큰 효과에도

Table 1. Determinants of the strength of recommendation

\begin{tabular}{|c|c|}
\hline Factor & Comment \\
\hline $\begin{array}{l}\text { Balance between desirable } \\
\text { and undesirable effects }\end{array}$ & $\begin{array}{l}\text { The larger the difference between the desirable and undesirable effects, the higher the likelihood that a strong } \\
\text { recommendation is warranted. The narrower the gradient, the higher the likelihood that a weak recommendation } \\
\text { is warranted. }\end{array}$ \\
\hline Quality of evidence & The higher the quality of evidence, the higher the likelihood that a strong recommendation is warranted. \\
\hline Values and preferences & $\begin{array}{l}\text { The more values and preferences vary, or the greater the uncertainty in values and preferences, the higher the } \\
\text { likelihood that a weak recommendation is warranted. }\end{array}$ \\
\hline Costs (resource allocation) & $\begin{array}{l}\text { The higher the costs of an intervention, that is, the greater the resources consumed. then the lower the likelihood } \\
\text { that a strong recommendation is warranted. }\end{array}$ \\
\hline
\end{tabular}

Adapted from the article of Guyatt et al. BMJ 2008;336:1049-51 [1]. 
불구하고 압박스타킹의 사용은 약한 권고로 결정되었습니다 [4].

세 번째 핵심 요소는 가치와 선호도의 불확실성(uncertainty) 혹은 다양성(variability)입니다. 중재는 항상 장단점 이 있으므로, 진료지침 개발 패널은 권고 강도를 결정할 때 중재의 이점, 위험 및 불편함을 고려하여야 합니다. 항혈전치 료를 하지 않는 것에 비하여 warfarin은 대략 $65 \%$ 정도 뇌졸 중의 위험도를 낮추지만 중증 위장관 출혈의 위험도가 증가합 니다. Devereaux 등[5]은 63명의 임상의사와 61 명의 환자에 게 warfarin의 뇌졸중 예방효과(이점)와 중증 위장관 출혈(위 험)에 대한 가치와 선호도에 대한 연구를 진행하였습니다. 임상의사의 경우 답변의 다양성을 보였지만, 대부분의 환자에 서 뇌졸중을 예방하는 데 높은 가치를 두었으며 $8 \%$ 의 뇌졸중 을 예방하기 위해 $22 \%$ 의 위장관 출혈의 위험도를 감수할 수 있다고 답변하였습니다. 환자의 가치와 선호도 역시 다양하 게 보고되었으나 몇몇 환자들은 단지 작은 위험도의 위장관 출혈만을 감수할 수 있다고 하였습니다. 이 연구에 따르면 warfarin 치료는 뇌졸중 고위험군 환자에서만 강한 권고를 할 수 있을 것입니다.

심부정맥혈전을 가진 임산부에서 warfarin 사용을 생각해 봅시다. 임신 6 주에서 12 주 사이의 warfarin 치료는 심각하지 는 않으나 태아 발달 이상을 초래할 위험이 있습니다. 대체 치료인 heparin은 태아에게 이러한 발달 이상을 초래하지 않는 것으로 알려져 있습니다. 그러나 heparin 투여는 통증과 불편감, 그리고 비용의 상승이라는 단점을 동반합니다. 임상 의사 입장에서 여성들은 '태아 합병증 발생의 예방'에 높은 가치를 둔다는 갖는다는 것은 명확합니다. 따라서 앞서 언급한 단점에도 불구하고 심부정맥혈전을 가진 임산부에서 heparin 대체 치료는 강한 권고가 될 수 있습니다.

마지막 핵심 요소는 비용입니다. 비용은 다른 요소에 비하여 시간과 지역에 따라 더 많은 다양성을 가질 수 있습니다. 특허 가 만료되면 약값이 폭락하는 경향이 있으며, 동일한 약물에 대한 비용은 지역마다 크게 다릅니다. 자원의 활용 역시 다양합 니다. 예를 들어 동일한 약물에 대해 연 처방 비용은 미국에서 한 명의 간호사 봉급에 해당할 수 있지만 중국에서는 30명의 간호사 봉급에 해당할 수 있습니다. 따라서 비용이 높다면 강한 권고의 가능성은 낮아지지만 권고 시 해당 의료 환경 (context of recommendation)에 대한 고려는 매우 중요합 니다. 자원 할당을 고려하면, 진료지침 개발 패널은 권고가 적용이 되는 해당 의료 상황을 반드시 고려하여야 합니다.

\section{강한 권고는 모든 경우에 중요한 것은 아닙니다.}

만약 선택 (치료)의 결과가 상대적으로 중요하지 않다면 일부 환자는 강한 권고에 대해서도 신경 쓰지 않을 수 있습니
다. 환자들이 자신의 생활방식을 바꾸어야 하는 새로운 많은 약제 또는 권고와 연관되어 있을 때 특히 그렇습니다. 우선 순위를 정할 때 정부와 공중 보건 관계자들은 또한 권고 강도 이외의 요소들을 고려해야 합니다. 이러한 요소들로는 특정 질병의 유병률, 형평성(equity)의 고려, 및 의료의 질 개선 가능성 등이 있으며 이는 전체 인구의 공중 보건 증진에 영향을 미칠 수 있는 것들입니다.

\section{연구 목적(Research Context)으로 중재를 사 용하라는 권고는 적절할 수 있습니다.}

진료지침 개발 패널은 이점의 근거가 부족하고 상당한 위해 성과 비용을 수반하는 유망한 새로운 중재에 대해 결정을 내려야 하는 경우도 있습니다. 이 경우 진료지침 개발 패널은 해당 중재의 임상적 사용을 금지하거나 또는 중재를 사용하도 록 부적절하게 약한 권고를 내리기 어려울 수 있습니다. 이 중재를 임상에서 사용하지 말라는 권고는 중재에 대한 추가적 인 연구를 제한할 수 있기 때문입니다. 연구의 목적으로만 치료를 사용하라는 권고가 이러한 문제를 완화시킬 수 있기 때문입니다. 더욱이 이러한 권고는 연구자들에게 중요한 연구 질문(research question)에 해답을 얻기 위한 노력을 북돋을 수 있을 것입니다.

\section{근거수준 및 권고 강도에 대한 다양한 표현은 적 절 할 수 있습니다.}

대부분의 진료지침 개발 패널은 그들의 권고를 요약하기 위해 단어와 숫자를 사용하지만 그 의미는 각각 다를 수 있습니 다. 이것은 우리를 매우 혼란스럽게 만듭니다[6]. 비록 임상의 사들이 권고를 표현하는 숫자와 단어에 익숙한 것과 같이 (언어 소통에 적합한) 여러 의료 협의체(organization)들이 글자와 숫자를 선택하는 것에 충분한 이유가 있다 할지라도 근거수준과 권고 강도에 대한 기호를 이용한 표현(symbolic

Table 2. Representations of quality of evidence and the strength of recommendations

\begin{tabular}{ll}
\hline Quality of evidence & $\oplus \oplus \oplus \oplus$ or A \\
High quality & $\oplus \oplus \oplus \bigcirc$ or B \\
Moderate quality & $\oplus \oplus \bigcirc \bigcirc$ or C \\
Low quality & $\oplus \bigcirc \bigcirc \bigcirc$ or D \\
Very low quality & \\
Strength of recommendation & $\uparrow \uparrow$ or 1 \\
Strong recommendation for using an intervention & $\uparrow ?$ or 2 \\
Weak recommendation for using an intervention & $\uparrow ?$ or 2 \\
Weak recommendation against using an intervention & $\downarrow ?$ or 1 \\
Strong recommendation against using an intervention & $\downarrow \downarrow$ or 1 \\
\hline Adapted from the article of Guyatt et al. BMJ 2008;336:1049-51 [1].
\end{tabular}


representation)은 이러한 점에서 자유로울 수 있습니다. GRADE는 근거수준과 권고 강도에 대해 선호되는 기호적 표현, 숫자 및 글자들을 제공합니다(Table 2 and Appendix 2) $[1,6]$.

\section{CONFLICT OF INTEREST}

No potential conflict of interest relevant to this article are reported.

\section{AUTHOR CONTRIBUTIONS}

E.C.H.: translating the article, and drafting the manuscript, D.K.K,, J.Y.K., H.J.J., H.W.K., and H.W.K.: helping to translate and draft the manuscript, J.H.J.: contacting BMJ editorial office to get the approval, helping to translate and draft the manuscript, and final approval.

\section{ORCID}

Eu Chang Hwang, https://orcid.org/0000-0002-2031-124X

Do Kyung Kim, https://orcid.org/0000-0002-3696-8756

Ho Won Kang, https://orcid.org/0000-0002-8164-4427

Ja Yoon Ku, https://orcid.org/0000-0003-3460-9386

Hyun Jin Jung, https://orcid.org/0000-0002-1895-7180
Hong Wook Kim, https://orcid.org/0000-0002-3847-1401 Jae Hung Jung, https://orcid.org/0000-0002-4990-7098

\section{REFERENCES}

1. Guyatt GH, Oxman AD, Kunz R, Falck-Ytter Y, Vist GE, Liberati A, et al.; GRADE Working Group. Going from evidence to recommendations. BMJ 2008;336:1049-51.

2. Fleisher LA, Bass EB, McKeown P; American College of Chest Physicians. Methodological approach: American College of Chest Physicians guidelines for the prevention and management of postoperative atrial fibrillation after cardiac surgery. Chest 2005;128(2 Suppl):17-23S.

3. O'Connor AM, Stacey D, Entwistle V, Llewellyn-Thomas $H$, Rovner D, Holmes-Rovner M, et al. Decision aids for people facing health treatment or screening decisions. Cochrane Database Syst Rev 2003;(2):CD001431.

4. Geerts W, Ray JG, Colwell CW, Bergqvist D, Pineo GF, Lassen $\mathrm{MR}$, et al. Prevention of venous thromboembolism. Chest 2005;128:3775-6.

5. Devereaux PJ, Anderson DR, Gardner MJ, Putnam W, Flowerdew G), Brownell BF, et al. Differences between perspectives of physicians and patients on anticoagulation in patients with atrial fibrillation: observational study. BMJ 323:1218-22.

6. Schunemann HJ, Best D, Vist G, Oxman AD; GRADE Working Group. Letters, numbers, symbols and words: how to communicate grades of evidence and recommendations. CMAJ 2003;169:677-80. 
Appendix 1. 권고 강도의 결정 요소

\section{요소}

바람직한 효과(desirable effects)와 바람직하지 않은 효과(undesirable effects)의 균형

근거수준(quality of evidence)

가치와 선호도

비용(resource allocation)
설명

바람직한 효과(desirable effects)와 바람직하지 않은 효과(undesirable effects)의 차이가 클수록 강한 권고를 내릴 가능성 이 높아짐. 차이가 작거나 효과에 대한 확신이 낮을수록 약한 권고를 내릴 가능성이 높아짐.

근거수준이 높을수록 강한 권고를 내릴 가능성이 높아짐.

가치와 선호도가 다양하거나 불확실성이 클수록 약한 권고를 내릴 가능성이 높아짐.

중재의 비용이 클수록 (자원 이용이 많을수록) 강한 권고가 내려질 가능성은 낮아짐.

Appendix 2. 근거수준과 권고 강도의 표현

\begin{tabular}{ll}
\hline 근거수준 & \\
높음 & $\oplus \oplus \oplus \oplus$ or A \\
중등도 & $\oplus \oplus \oplus \bigcirc$ or B \\
낮음 & $\oplus \oplus \bigcirc \bigcirc$ or C \\
매우 낮음 & $\oplus \bigcirc \bigcirc \bigcirc$ or D \\
권고 강도 & \\
중재 사용에 대한 강한 권고 & $\uparrow \uparrow$ or 1 \\
중재 사용에 대한 약한 권고 & $\uparrow ?$ or 2 \\
중재 사용 반대에 대한 약한 권고 & $\downarrow ?$ or 2 \\
중재 사용 반대에 대한 강한 권고 & $\downarrow \downarrow$ or 1 \\
\hline
\end{tabular}

